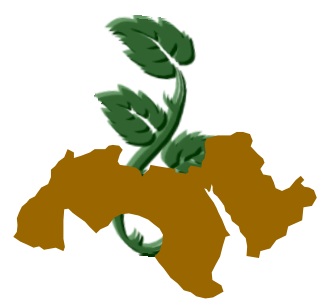

Arab Univ.

\title{
DETECTING HOLLOW HEART OF POTATO TUBERS USING IMPACT SOUND
}

\author{
Elbatawi ${ }^{1}$, I.E. and M.S. Omran ${ }^{2}$ \\ 1- Agricultural Engineering Research Institute, P. O. Box 256, Nadi Elsaid St., Dokki, Giza, \\ Egypt \\ 2- Agricultural Engineering Department, Faculty of Agriculture, Cairo University, Cairo, Egypt
}

Keywords: Acoustic impact method, Potato tubers, Resonant frequency, Hollow heart, Cavity

\begin{abstract}
An acoustical sorting system was developed to detect hollow heart (cavity) of potato tubers (Spunta variety). The system includes a microphone and digital signal processing hardware. It was found that upon impact with a steel plate, potato tubers with no hollow heart emit sound with higher signal magnitudes than with hollow one. Linear discriminate analysis was used to classify potato tubers using three features extracted from the microphone signal. One of the discriminate features is the integrated absolute value of microphone output signal. The other two features are the number of data points in the digitized microphone signal after impact that have a slope and signal magnitude below preset threshold levels. The classification accuracy of this system is approximately $98 \%$. The internal quality of potato tubers can be detected by acoustic impact method. The resonant frequency of potato tubers after dropping was found from acquired signal. The hollow heart existence of potato tubers can be evaluated by the resonant frequency of vibration signal of dropping tubers. This technology of acoustics to evaluate the hollow heart existence is more reliable and accurate specially for export.
\end{abstract}

\section{INTRODUCTION}

Egypt produces $1.8 \sim 2.0$ million ton of potato every year and Spunta variety is one of the mostly used tubers by consumers (Agric. Res. Center Bulletin, 2005). The hollow heart (cavity) in potato tubers is actually due to the excess of nitrogen fertilizer during growing specially for bigger tubers such as Spunta variety. Both hollow and black heart are not seen by human eyes and there is no way to detect them. Some researches have been done on watermelon quality, pistachio nut shell and egg shell using acoustic impact methods but there is no research on hollow heart of potato tubers. Brown center and hollow heart are physiological disorders of the potato tuber and arise at a higher incidence when growing conditions abruptly change during the season. This can arise when the potato plants recover too quickly after a period of environmental or nutritional stress. When the tubers begin to grow rapidly, the tuber pith can die and/or pull apart leaving a void in the center (Hochmuth et al 2001). Hollow heart is characterized by a star shape hollow (cavity) in the center of the tuber on the other hand, brown center frequently precedes the development of hollow heart. Hollow heart and brown center negatively impact tuber quality. Disorders make the tubers unattractive and can reduce repeat sales but severe hollow heart negatively impacts the quality of chip processing potatoes. However, both disorders are reported as not harmful and do not effect the taste or nutrition of the tuber. USDA has established grade and quality standards and recommendation for potatoes to help potato growers and shippers market their product in wholesale channels.

Most farmers can not predict the internal quality of potato tubers at all. From this viewpoint, Takeda et al (1970) made an instrument for nondestructive evaluation of apple ripeness by measuring sound when the product was hit by impact. The technology of analyzing the sound was to find a relationship between natural frequency and maturity of the product. Clark (1975) also used the same idea to predict the ripeness of watermelons. Chyung 
(1997) investigated the internal qualities of watermelon, such as maturity, cavity existence and orientation, using acoustics by impulse striking. The watermelon was struck by a hammer and the produced sound was acquired by a directional microphone and signal analyzer to find out the relationship between peak frequency and quality. Several different acoustic methods to evaluate food quality have been investigated. McCambridge et al (1996) developed an acoustic method to detect freeze cracking in different foods during freezing. This study found that when cracks occur they produce sounds of higher amplitude and frequency than background noise. Pearson (2001) developed an acoustical sorting system to separate pistachio nuts with closed shells from those with open shells. He concluded that, the improved sorting accuracy of nuts allows $4.6 \%$ more open-shell product to be classified as open shell. On the other hand, Cetin et al (2004) developed an algorithm using speech recognition technology to distinguish pistachio nuts with closed shells from those with open shells. They observed that upon impact, nuts with closed shells emit different sounds than nuts with open shells and the classification accuracy was more than $99 \%$ on the validation set.

Armstrong et al (1990) mentioned that flesh firmness is used as an indicator of apple texture or maturity. Technique, as suggested by USDA (1978), for firmness determination includes Magness-Taylor firmness testing, thumb pressure, wax development, cutting and chewing test. Yamamoto et al (1980) compared Magness-Taylor firmness with apple resonant frequencies when excited by this method. Several researchers have found an inverse correlation between fruit firmness and resonant frequency (Duprat et al 1997 and Stone et al 1998). Most of the acoustical systems developed was tapping the food with a plunger, recording the resulting sound, then digitally processing the microphone signal to extract dominant frequency bands or other signal features correlated with firmness. Younce and Davis (1995) developed such a system to measure firmness of cherries using impact acoustics. Sugiyama et al (1998) developed an acoustical firmness tester for melons that measured sound transmission velocity. This technique eliminated some error caused by size and shape variations in the fruits.

The objective of this work was to investigate the feasibility of using impact sound as a means to detect hollow heart of Spunta potato tubers for high quality product export.

\section{MATERIALS AND METHODS}

Potato tubers (Spunta variety) were collected from the local market according to their size and common used. One hundred tubers were chosen depending on their outer appearances from the local market of Giza, Egypt. The sizes of potato tubers were almost the same.

\section{System design}

The system was designed to drop potato tubers to an impact surface, acquire the sound signal upon impact, process the data, and then divert the product into either with hollow heart or without hollow heart (Fig. 1). The roller conveyer was constructed of polished stainless steel $(3.0 \mathrm{~mm}$ thickness) to form a trough declining to an impact container. The bottom of the container was covered with a $2.0 \mathrm{~mm}$ thickness of a sponge cushion to reduce potato bruising during impact. The impact container was made of $30 \times 30 \mathrm{~mm}$ polished stainless steel bar. Large thickness was required to minimize vibration of the bar when impacted by a potato tubers. A highly directional microphone (MS45 and K9 powering module, Osaka Electronics Corporation) was used to minimize the effect of ambient noise and the external noise effect was controlled by amplifier. Output of the microphone was connected to a digital signal processor (DSP) card hosted in a personal computer (Pentium 3 processor, $164 \mathrm{MHz}$, Windows 2000 operating system). When a potato tuber impacted the container, the microphone output signal ranged from 0 to $1.0 \mathrm{~V}$. Data acquisition began when the microphone output rose above $0.05 \mathrm{~V}$.

\section{Signal processing and resonant frequency measurement}

Data points (features) used were limited to those that could be computed in real time with a sorting rate of one tuber/s. Sorting at this rate required a minimal lag time (5 seconds) between obtaining the data to make a decision and taking the dropped tuber out of the container. Thus, Features were extracted from either the absolute value of the signal level (signal magnitude), the absolute value of the signal gradient, or both. Signal gradient was computed from the following equation:

$G_{x}=\left|I_{(x-g a p)}-I_{(x+g a p)}\right|$

where $\mathbf{G}_{\mathbf{x}}$ is the signal gradient value at data point $\mathbf{x}, \mathbf{I}_{\mathbf{x}}$ is the signal level value at location $\mathbf{x}$ and gap is the interval between data points. 


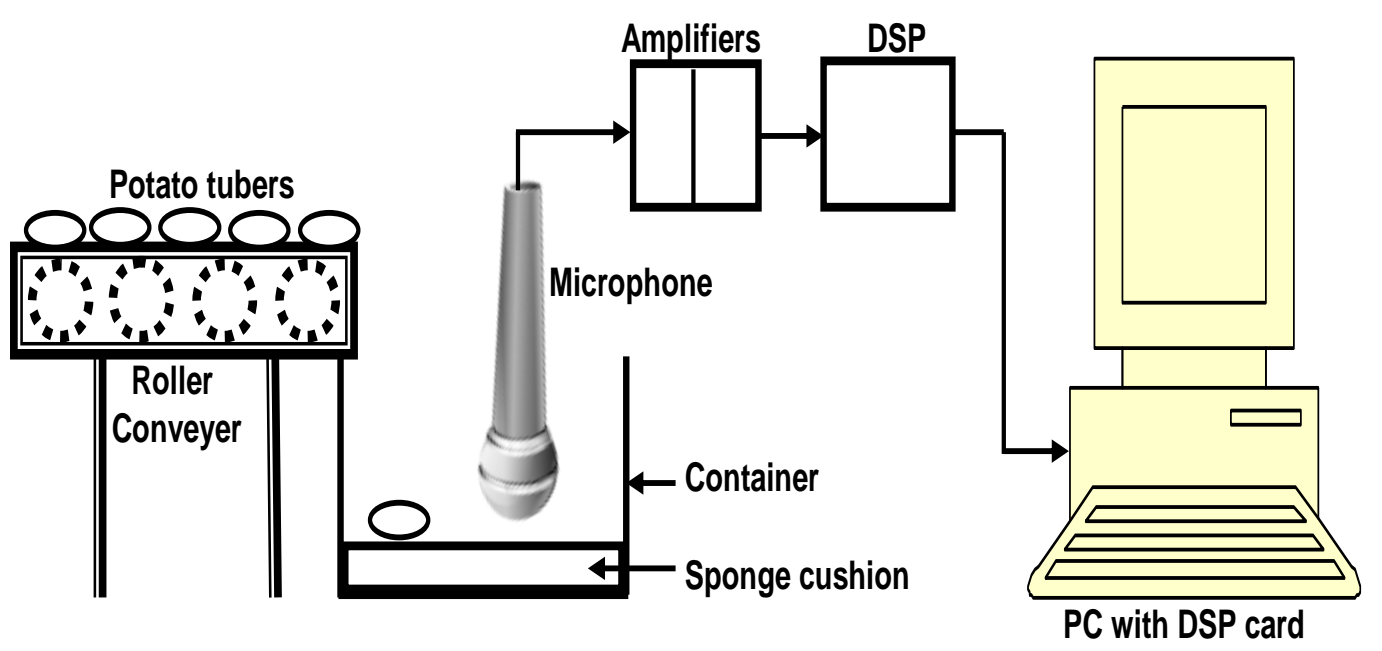

Fig. 1. Schematic diagram of the experimental and impact sound sorting system

The tuber was placed on a damping cushion and only direct sound waves were produced by dropping without reflection. The directional microphone was hanging in the container in order to enable the microphone to measure directly the sound of the dropped tubers. The directional microphone was connected to a signal analyzer (B\&K 1800 Dual Channel). The acquired signal, on the time and frequency (Fast Fourier Transform, FFT) domain, were shown on the signal analyzer panel and saved into disk. Potato tubers were divided into the categories of hollow inside and no hollow inside. The hollow in the tuber directly influences the resonant frequencies and the acoustic response was acquired by a directional microphone. First of all the consistency of the signal was confirmed.

\section{Cavity existence measurement}

To find the hollow, some marks were made on each tuber around the equator and cross section lines. The marks were divided into 4 parts by each $90^{\circ}$ along circumference of the tuber. The tuber was dropped on each mark (twice on the hip side and twice on the sharp side) and the microphone was placed on the opposite side of it by taking the tuber out of the container and dropping it again so the main peak frequency was obtained. After dropping the tuber four times and recording its data, the tuber was cut into two halves to see by human eye that the tuber has a hollow (cavity) or not. This way makes us sure about the existence of hollow heart or not and compare human eye results with the experimental data. Each mark was separated 90 angular degree on each tuber circumference. When a tuber was dropped at some specified marks, the main peak splits up in two or three different peaks. Each response was collected every $90^{\circ}$ of rotation about its longitudinal axis. A total of 360 data points (features) were computed for each tuber. However, a maximum of four of these data points could be computed in real time with the installed DSP hardware. Using all possible combinations of two, three, and four data points, a program "C" was written to exhaustively search for the best subset of features for classifying tubers as with or without hollow. Both linear and non-linear discriminant analyses were used as the classification procedure (Huberty 1994).

\section{RESULTS AND DISCUSSION}

\section{Cavity existence}

Table (1) shows some typical data of main peak frequencies for some potato tubers from the local market in Giza, Egypt. The multivariate discriminant analysis procedure in SAS (1990) was utilized to find the detection criteria among the three locations. The analysis showed that the main peak frequencies stayed almost at a constant level. On the other hand, there was a differences of peak frequencies between tubers with hollow heart and tubers without hollow heart. The average peak frequency was $1.157 \mathrm{kHz}$ for the tubers without hollow heart and average peak frequency was $0.957 \mathrm{kHz}$ for the tubers with hollow heart. The 
analysis also indicated that average peak frequency differences between hollow tubers and no hollow ones were about $230 \mathrm{~Hz}$ with SEP (Standard Error of Prediction) of 0.019 . From experimental data analysis, it was found that, peak frequency of hollow tubers is lower than that of no hollow. There is a range of about $201 \mathrm{~Hz}$ to distinguish from hollow (cavity) existence. Classification accuracy of the acoustic method was greatly improved by increasing the number of training sounds.

Table1. Some main peak frequencies of detecting hollow heart tubers

\begin{tabular}{|c|c|}
\hline $\begin{array}{c}\text { Without hollow heart } \\
\text { Freq. (kHz) }\end{array}$ & $\begin{array}{c}\text { With hollow heart } \\
\text { Freq. (kHz) }\end{array}$ \\
\hline 1.21 & 0.98 \\
1.15 & 0.96 \\
1.17 & 0.98 \\
1.18 & 0.95 \\
1.16 & 0.96 \\
1.16 & 0.94 \\
1.15 & 0.97 \\
1.14 & 0.95 \\
1.16 & 0.96 \\
1.12 & 0.94 \\
1.20 & 0.93 \\
1.14 & 0.94 \\
1.15 & 0.95 \\
1.14 & 0.94 \\
1.16 & 0.96 \\
1.15 & 0.98 \\
1.15 & 0.94 \\
1.14 & 0.94 \\
1.15 & 0.95 \\
1.14 & 0.97 \\
1.14 & 0.97 \\
1.17 & 0.98 \\
1.17 & 0.97 \\
1.18 & 0.95 \\
& \\
\hline
\end{tabular}

Only two out of 100 tubers were misclassified in all cases which represent more than $98 \%$ accuracy. It is however possible to detect the hollow existence using acoustic technology. The potato tuber was placed in the container after impact and the acoustic tests were repeated four times. Using discriminate analysis, it was desired to extract a limited number of features from the digitized microphone signal to accurately classify tubers as either with or without hollow heart. The correlation between the resonant frequencies detected in the positions was very high $(R=0.97)$.

Fig. (2) shows the normalized spectral amplitude obtained from the impact sound of Spunta potato tubers (with or without hollow). For this plot, frequency spectra for individual tuber was computed using a Hanning window (Brigham, 1988) on the first 256 data points sampled after impact then, the spectra was averaged. The frequency of sounds emanating from potato tubers (with or without hollow) was slightly different. Hollow heart tubers exhibited a peak near $3000 \mathrm{~Hz}$, while undamaged tuber (without hollow) have high normalized amplitude. Thus, the extracted features used to distinguish potato tubers split types relied heavily on the signal magnitude or a combination of the signal magnitude and gradient.

\section{Variation of acoustic signal with tuber position}

To have a visible signal, an offset of 0.2 relative amplitude units were added and two resonant frequencies of different amplitudes could be detected depending on the tuber location (Fig. 3). It was concluded that consistent readings can be achieved within a limited range of tuber position, despite its irregular shape.

The accuracy and repeatability of the piezoelectric measurement system was tested with the 100 tubers. The axis symmetrical tubers were dropped in the container and tested simultaneously by three sensors. Consistent frequency response was obtained when repeated tests were conducted at the same location on the tuber, this consistency was evident even with irregular acoustic signals. Small variations of the natural frequencies, in steps of $11.9 \mathrm{~Hz}$, could be detected while the tuber was successively rotated relative to its axis of symmetry. It was concluded that the acoustic test is repeatable and that accuracy of $11.9 \mathrm{~Hz}$ can be obtained when measuring the natural frequencies of a tuber.

\section{Area of power spectrum}

Fig. (4) shows the relationship between the average area under the power spectrum for all data sets of 100 Spunta potato tubers and the difference between maximum and minimum area. The maximum and minimum area differences for the normal tubers (no hollow) were mostly higher than those of with hollow tubers. This was due to the higher magnitude in power spectrum of the normal tubers than that of with hollow tubers as shown 


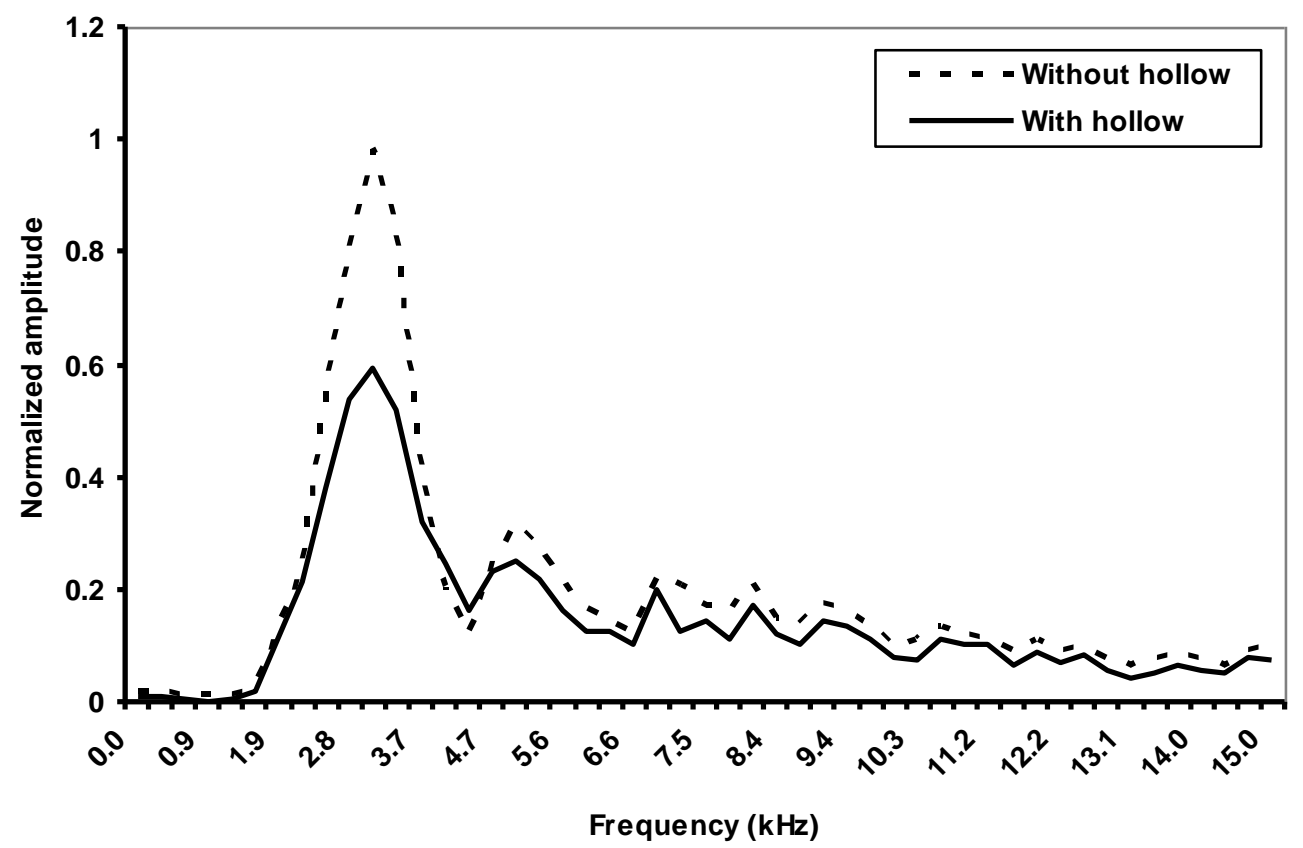

Fig. 2. Average frequency spectra magnitudes of the impact sound of Spunta potato tuber with and without hollow heart

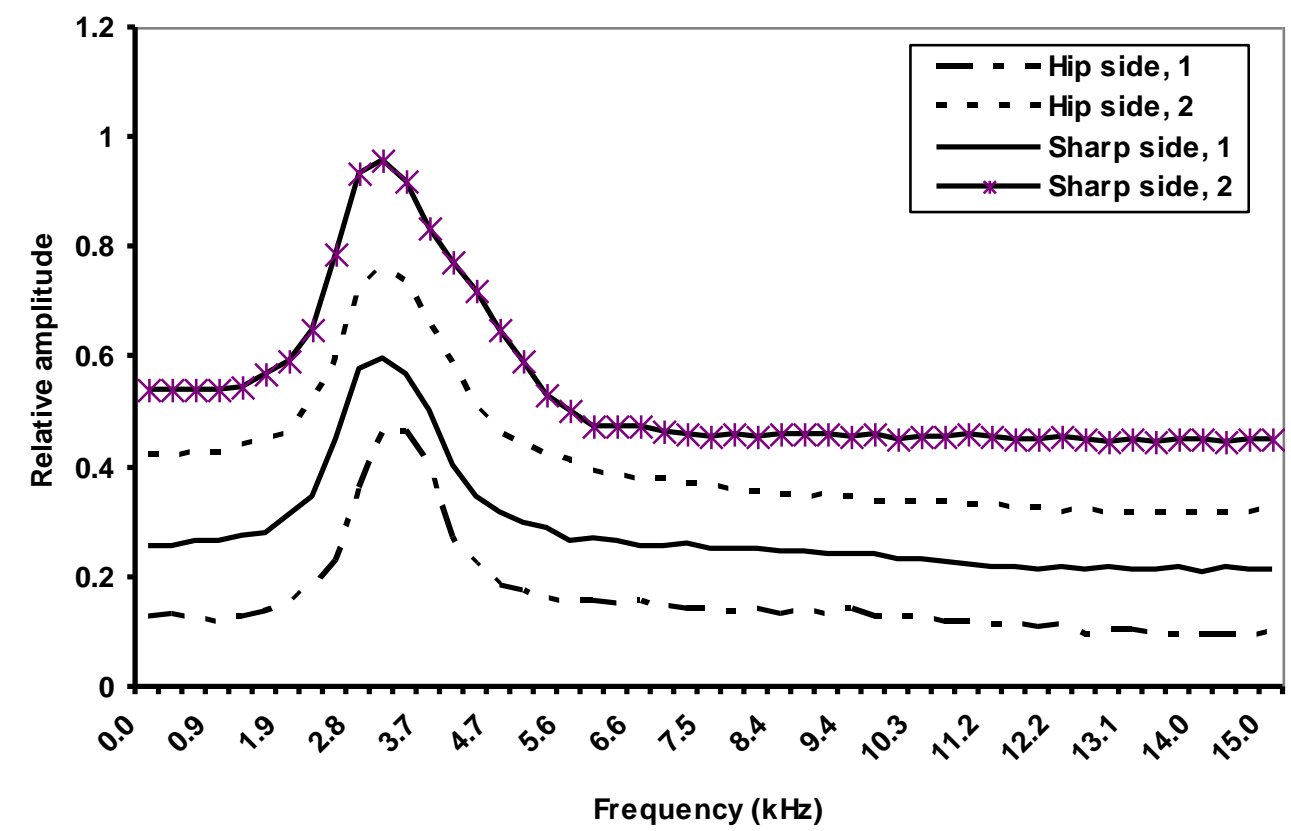

Fig. 3. Effect of fruit position on the acoustic signal and resonant peaks 


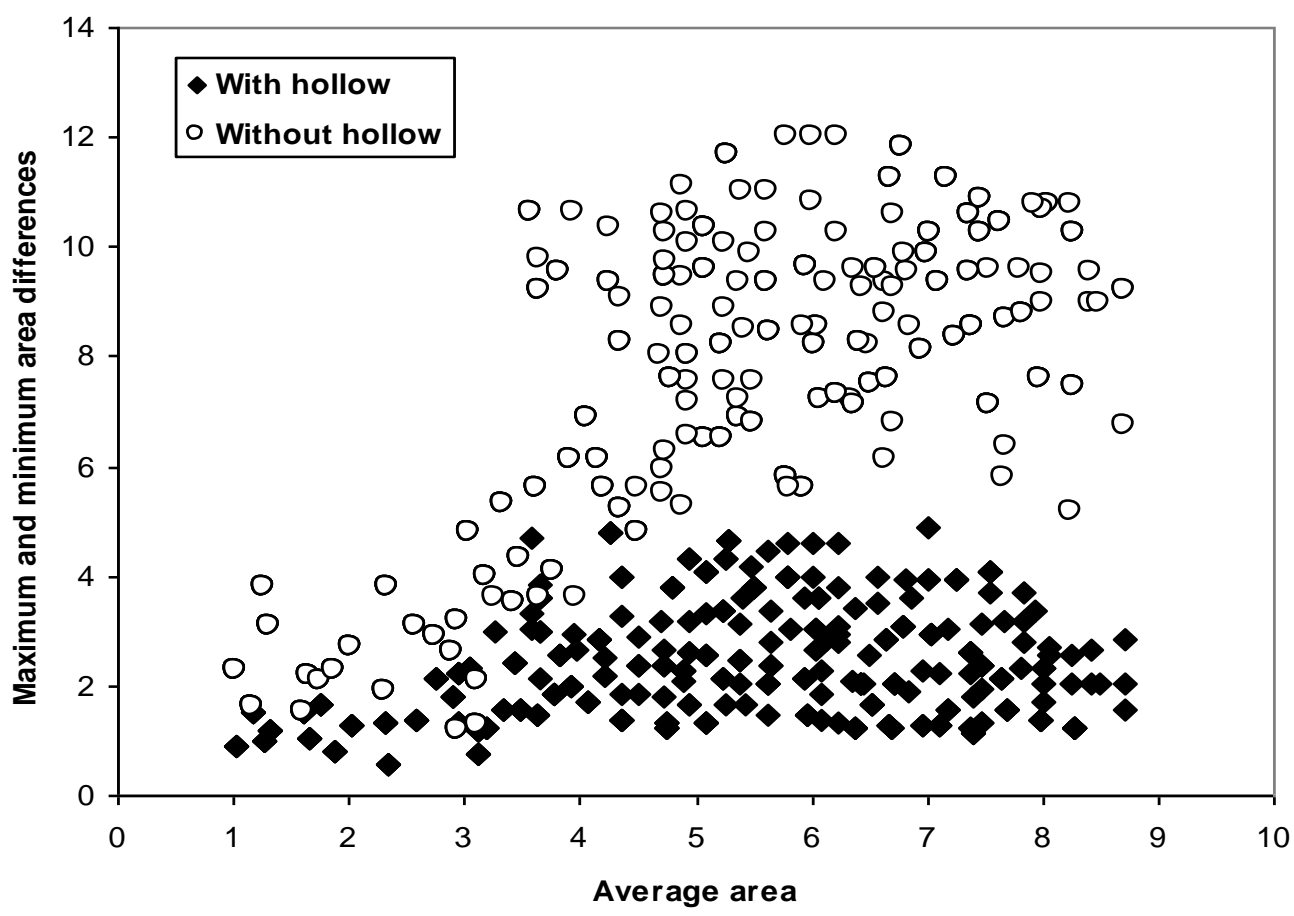

Fig. 4. Average area and difference between maximum and minimum area in normalized power spectrum curve for hollow and no hollow tubers

before in Fig. (2). The differences in maximum and minimum areas for normal tubers were higher than those of with hollow tubers because the four sound responses (two hip sides and two sharp sides) from a normal tubers were more uniform than those of with hollow tubers. The criteria to distinguish hollow tubers could be drawn from the average area or differences between maximum and minimum areas or both of them.

\section{CONCLUSION}

The hollow heart existence and orientation of potato tubers can be evaluated by resonant frequencies of acoustic signal through an impulse vibration. This study showed the feasibility of distinguishing the tubers with and without hollow (cavity) heart by impact acoustics. The criteria to distinguish hollow tubers could be drawn from the average area or differences between maximum and minimum areas or both of them. The correlation between the resonant frequencies detected in the positions was very high $(R=0.97)$ and a lower correlation was found between the damping ratio $(R=0.65)$. Only two out of 100 tubers were misclassified in all cases which represent more than $98 \%$ of accuracy. No conclusion has been found yet about the preferred tuber position in this case.

\section{REFERENCES}

Agricultural Research Center (2005). Production and Storage of Potato Tubers in Egypt. Ministry of Agriculture, Horticulture Research Institute, A.R.C. Bulletin No. 9: 13-25.

Armstrong, P.; H.R. Zapp and G.K. Brown (1990). Impulsive excitation of acoustic vibrations in apples firmness determination. Transaction of ASAE 33(4): 1353-1359.

Brigham, O.E. (1988). The Fast Fourier Transform and its Applications. pp. 123-209. Prentice Hall, Englewood Cliffs, N. J., U.S.A.

Cetin, A.E.; T.C. Pearson and A.H. Tewfik (2004). Classification of closed and open shell pistachio nuts using voice recognition technology. Transactions of the ASAE 47(2): 659-664.

Chyung, A. (1997). Acoustic Evaluation of Watermelon Internal Quality - Maturity and Cavity Existence and Orientation. Proceedings of international symposium on agricultural mechanization and automation. Taipei, Taiwan, 2: 239-244.

Clark, R.L. (1975). An investigation of the acoustical properties of watermelon as related to maturity. ASAE Paper No. 75-6004, Amer. Soc. Agric. Eng., St. Joseph, Mich., U.S.A. 
Duprat, F.; M. Grotte; E. Pietri and D. Loonis (1997). The Acoustic Impulse Response Method for Measuring the Overall Firmness of Fruit. Journal of Agricultural Engineering Research, 66(4): 251-259.

Hochmuth, G.J.; C.M. Hutchinson; D.N. Maynard; W.M. Stall; T.A. Kucharek; S.E. Webb; T.G. Taylor; S.A. Smith and E.H. Simonne (2001). Potato Production in Florida. In: Vegetable Production Guide for Florida. pp. 168-213. Edited by; Maynard, D.N. and S.M. Olson. Vance Publishing, U.S.A.

Huberty, C.J. (1994). Applied Discriminant Analysis. pp. 233-266. John Wiley and Sons Inc., New York, U.S.A.

McCambridge, M.A.; Y.C. Hung and P. Mallikarjunan (1996). Detection of Freeze-Crack using Digital Signal Processing. Applied Engineering in Agriculture 12(4): 481-485.

Pearson, T.C. (2001). Detecting of Pistachio Nuts with Closed Shells using Impact Acoustics. Applied Engineering in Agriculture 17(2): 249-253. SAS/STAT User Guide (1990). Ver. 6, $4^{\text {th }}$ Ed., Vol. 2. Statistical Analysis System Institute, Inc. Cary, N.C., U.S.A.
Stone. M.L.; P.R. Armstrong; D.D. Chen; G.H. Brusewitz and N.O. Maness (1998). Peach Firmness Prediction by Multiple Location Impulse Testing. Transactions of the ASAE, 41(1): 115-119. Sugiyama. J.; T. Katsurai; J. Hong; H. Koyama and K. Mikuriya (1998). Melon ripeness monitoring by a portable firmness tester. Transactions of the ASAE 41(1): 121-127.

Takeda, Y.; M. Sawaji and I. Yasukawa (1970). The non-destructive measurement of ripeness of apples by the sonic characteristics. Nippon Shokukin Kogyo Gakkaishi (Japan) 17(1): 351-360. (Japanese).

USDA, (1978). APPLES, Shipping Point Inspection Instructions, Fruit and Vegetable Division, Fresh Products Branch, Washington, D.C., U.S.A. Yamamoto, H.; M. Iwamoto and S. Haginuma (1980). Acoustic Impulse Response Method for Measuring Natural Frequency of Intact Fruits and Preliminary Applications to Internal Quality Evaluations of Apples and Watermelons. Journal of Texture Studies, 11: 117-136.

Younce, F.L. and D.C. Davis (1995). A Dynamic Sensor for Cherry Firmness. Transactions of the ASAE, 38(5): 1467-1476. 